\title{
The Shortest Isoform of Dystrophin (Dp40) Interacts with a Group of Presynaptic Proteins to Form a Presumptive Novel Complex in the Mouse Brain
}

\author{
Takenori Tozawa • Kyoko Itoh • Takeshi Yaoi • \\ So Tando • Masafumi Umekage • Hongmei Dai • \\ Hajime Hosoi • Shinji Fushiki
}

Received: 8 November 2011 / Accepted: 2 January 2012 / Published online: 19 January 2012

(C) The Author(s) 2012. This article is published with open access at Springerlink.com

\begin{abstract}
Duchenne muscular dystrophy (DMD) causes cognitive impairment in one third of the patients, although the underlying mechanisms remain to be elucidated. Recent studies showed that mutations in the distal part of the dystrophin gene correlate well with the cognitive impairment in DMD patients, which is attributed to Dp71. The study on the expression of the shortest isoform, Dp40, has not been possible due to the lack of an isoform specific antibody. Dp40 has the same promoter as that found in Dp71 and lacks the normal C-terminal end of Dp427. In the present study, we have raised polyclonal antibody against the $\mathrm{N}$-terminal sequence common to short isoforms of dystrophin, including Dp40, and investigated the expression pattern of Dp40 in the mouse brain. Affinity chromatography with this antibody and the consecutive LC-MS/MS analysis on the interacting proteins revealed that Dp40 was abundantly expressed in synaptic vesicles and interacted with a group of presynaptic proteins, including syntaxin1A
\end{abstract}

Electronic supplementary material The online version of this article (doi:10.1007/s12035-012-8233-5) contains supplementary material, which is available to authorized users.

T. Tozawa $\cdot$ K. Itoh $(\bowtie) \cdot$ T. Yaoi $\cdot$ S. Tando $\cdot$ M. Umekage $\cdot$

H. Dai $\cdot$ S. Fushiki

Department of Pathology and Applied Neurobiology,

Graduate School of Medical Science,

Kyoto Prefectural University of Medicine,

465 Kajii-cho, Kawaramachi Hirokoji,

Kamigyo-ku, Kyoto 602-8566, Japan

e-mail: kxi14@koto.kpu-m.ac.jp

T. Tozawa $\cdot$ H. Hosoi

Department of Pediatrics, Graduate School of Medical Science,

Kyoto Prefectural University of Medicine,

465 Kajii-cho, Kawaramachi Hirokoji,

Kamigyo-ku, Kyoto 602-8566, Japan and SNAP25, which are involved in exocytosis of synaptic vesicles in neurons. We thus suggest that Dp40 may form a novel protein complex and play a crucial role in presynaptic function. Further studies on these aspects of Dp40 function might provide more insight into the molecular mechanisms of cognitive impairment found in patients with DMD.

Keywords Duchenne muscular dystrophy (DMD) - Brain . Dystrophin · Dp40 $\cdot$ SNARE $\cdot$ Presynapse

\section{Introduction}

Duchenne muscular dystrophy (DMD) is an X-linked hereditary disease characterized by progressive muscular degeneration. It is caused by mutations in the gene encoding dystrophin that result in either the absence or the dysfunction of dystrophin, a 427-kDa protein localized in the plasma membrane of muscle cells [1]. In addition, about one third of DMD patients manifest variable degrees of cognitive impairment, which is not progressive in nature and does not correlate with the severity of the muscular disease [1-3]. A detailed neuropsychological study showed that children with DMD have specific types of learning disabilities, exhibiting significantly lower scores in verbal memory and delays in reading and learning [4]. Although the brain is the organ showing most abundant expression of dystrophin, next to muscle [5], the functions of dystrophin in the brain remain to be elucidated. Two alternative $427-\mathrm{kDa}$ dystrophin isoforms, originating from two distinct proximal promoters, are expressed in the cerebral cortex and in the cerebellum $[2,3]$ : two smaller alternatively spliced C-terminal products, $140-\mathrm{kDa}$ dystrophin (Dp140) and 71-kDa dystrophin (Dp71) [6]. The transcription of 
Dp140 is initiated upstream from exon 45, and that of Dp71 is from between exon 62 and exon 63 [7]. Previous studies indicated that point mutations in the Dp71 coding region were closely associated with cognitive impairment observed in DMD patients [8-11].

Dp40 is the shortest isoform of dystrophin identified by sequencing of a cDNA clone isolated from a rat Schwannoma library [12] (Fig. 1). The 5'-UTR and the sequence for the first seven amino acids are identical to that of the 4.8-kb mRNA encoding Dp71, but the 3'-UTR is derived from the intron 70 sequence. Dp40 is unique since it lacks the normal C-terminal end of Dp427 (amino acids 3409-3685) [7]. Since Dp40 has the same promoter as that of Dp71, the expression of Dp40 might be affected as well in DMD patients with mutations located in Dp71. Daoud et al. reported that most of DMD patients with severe mental retardation have mutations in exon 63-69 which influenced the expression of both Dp71 and Dp40 [10]. As Dp40 is devoid of the common C-terminal regions, it is expected that antibodies against common $\mathrm{C}$-terminal regions of all dystrophin isoforms are unable to recognize Dp40.

In order to understand the presumptive function of Dp40 in the brain, we have investigated what proteins interact with Dp40 by using the novel antibody that we raised, which enabled us to recognize the first 15 amino acids, i.e., N-terminal sequences common to both Dp71 and Dp40. The affinity chromatography and LC-MS/MS analysis revealed that Dp40 interacted with a group of presynaptic proteins, including syntaxin1A, SNAP25, and VAMP2, so far known to be involved in exocytosis of synaptic vesicles in neurons [13-15]. These lines of evidence suggest that Dp40 might play a crucial role in presynaptic functions in the brain.

\section{Material and Methods}

Animals

Eight-week-old male ICR mice were purchased from a breeder (SHIMIZU Laboratory Supplies Co., Ltd., Kyoto, Japan). All animal studies were approved by the Institutional Review Board for Biomedical Research using Animals at Kyoto Prefectural University of Medicine, and the animals were handled according to the institutional guidelines and regulations.

Antibodies

N-terminal Specific Antibody Against Short Isoforms of Dystrophin

A polyclonal antibody against a synthetic peptide (MREQL KGHETQTTCW, corresponding to the first 15 amino acids of Dp71 N-terminus: Dp71N) was raised in rabbit with a conventional protocol (Iwaki, Japan). We will refer to this antibody as N-terminal specific antibody against short isoforms of dystrophin (N-term $\mathrm{Ab}$ ). In order to confirm the specificity of the N-term $\mathrm{Ab}$, we used recombinant proteins containing glutathione-S-transferase (GST) fused to Dp71N expressed in Escherichia coli BL21 as well as the insect cell line-expressed recombinant mouse Dp40 (kind gift of Prof. H. Mori). Next, we attempted to detect the three short isoforms of dystrophin in adult mouse whole brain lysate by western blot. Finally, we confirmed whether this antibody was applicable to the immunoprecipitation of the short isoform proteins by using the aforementioned GST-fused protein.

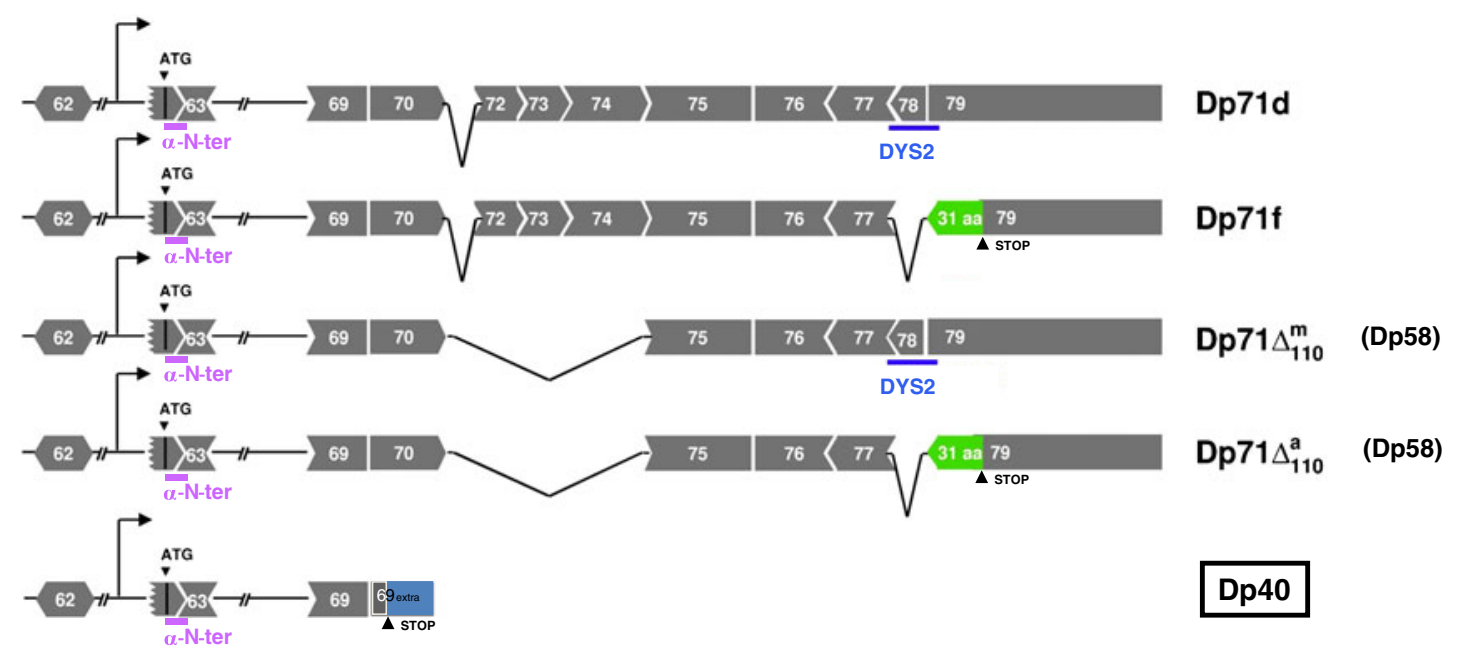

Fig. 1 The smaller alternatively spliced C-terminal products, 71-kDa dystrophin (Dp71), 58-kDa dystrophin (Dp71 $\Delta 110$; Dp58) and Dp40, and the epitopes which the C-terminal anti-dystrophin antibody (Dys2) and N-term Ab react are shown. Dp40 is the shortest isoform of dystrophin, and the sequence for the first seven amino acids is identical to that of the $4.8-\mathrm{kb}$ mRNA encoding Dp71, but lacks the normal C-terminal end of Dp427 (amino acids 3409-3685) 


\section{Other Primary Antibodies}

A rabbit polyclonal antibody against syntaxin1A (ab41453, abcam, MA, USA), a rabbit polyclonal antibody against SNAP25 (ab5666, abcam, MA, USA), a rabbit polyclonal antibody against VAMP2 (ab3347, abcam, MA, USA), a mouse monoclonal antibody against SNAP25(SP12) (ab24732, abcam, MA, USA), a mouse monoclonal antibody against VAMP 1/2 (SP10) (sc-20039, Santa Cruz Biotechnology, CA. USA ), a mouse monoclonal antibody against syntaxin1 (HPC-1) (sc-12736, Santa Cruz Biotechnology, CA. USA), a mouse monoclonal antibody against synaptotagmin (MAB5202, CHEMICON/ Millipore, CA, USA), and a mouse monoclonal antibody against CaMK II alpha (6 G9) (ab5683, abcam, MA, USA) were used throughout the present study.

\section{Secondary Antibodies}

We employed anti-rabbit immunoglobulin antibodies conjugated to horseradish peroxidase and anti-mouse immunoglobulin antibodies conjugated to horseradish peroxidase (GE Healthcare, UK), rabbit IgG TrueBlot ${ }^{\mathrm{TM}}$ HRP and mouse rabbit IgG TrueBlot ${ }^{\mathrm{TM}}$ HRP (eBioscience, San Diego, CA, USA), AlexaFluor 647 goat anti-rabbit IgG, AlexaFluor 488 goat anti-mouse $\mathrm{IgG}$, and AlexaFluor ${ }^{\mathbb{B}} 488$ goat anti-mouse IgM (Invitrogen, Japan).

\section{Immunoblot of Total Protein Extracts from Mouse Brain}

Total protein extracts from various brain regions of the mouse brain (cerebral cortex, hippocampus, thalamus, cerebellum) were prepared by homogenizing in the extraction buffer ( $2 \%$ sodium dodecyl sulfate (SDS), 62.5-mM Tris- $\mathrm{HCl}, \mathrm{pH} 6.8$, $5 \% \beta$-mercaptoethanol, $10 \%$ glycerol). One hundred $\mu \mathrm{g}$ of total extracted protein from each tissue was separated by SDSPAGE and transferred to polyvinylidene difluoride (PVDF) membrane. After blocking with 5\% skim milk in TBS (20$\mathrm{mM}$ Tris, $\mathrm{pH} 7.5,150-\mathrm{mM} \mathrm{NaCl} / 0.05 \%$ Tween-20), the membrane was incubated with $\mathrm{N}$-term Ab (dilution 1:1,000) for $1 \mathrm{~h}$ at room temperature (RT). Blots were incubated for $1 \mathrm{~h}$ at RT with anti-rabbit antibodies conjugated to horseradish peroxidase (dilution 1:1,000). Chemiluminescence was detected using Super signal West pico (PIRECE, USA) and BioMax XAR film (Kodak, Rochester NY, USA).

Immunohistochemistry of Mouse Brain and Muscle

Adult mice were transcardially perfused with $0.1-\mathrm{M}$ phosphate-buffered saline ( $\mathrm{pH}$ 7.4, PBS). The brain and gastrocnemius muscle were rapidly dissected; $10-\mu \mathrm{m}$-thick frozen sections were prepared using cryomicrotome (Leica, Wetzlar, Germany), mounted onto glass slides and fixed with $2 \%$ paraformaldehyde for 5 min. After blocking, they were incubated with $\mathrm{N}$-term $\mathrm{Ab}$ (dilution; 1:1,000), followed by FITC-conjugated goat anti-rabbit IgG (dilution; $1: 1,000)$ as secondary antibody. Sections were observed under a fluorescence microscope (Nikon, Eclipse 1000, Japan).

\section{Subcellular Fractionation}

The cerebral cortex and the hippocampus from 20 mice were homogenized and fractionated as previously described [16]. Briefly, brains were homogenized in buffered sucrose (320-mM sucrose, 4-mM HEPES-KOH, pH 7.4) supplemented with protease inhibitors. The homogenate was centrifuged for $4 \mathrm{~min}$ at $3,340 \mathrm{rpm}(1,100 \mathrm{~g})$. The resulting pellet (P1) was stocked, while the supernatant (S1) was collected and centrifuged for $4 \mathrm{~min}$ at 9,660 rpm $(9,200 \mathrm{~g})$. The supernatant (S2) was pooled, and the pellet (P2) was washed by re-suspending in buffered sucrose and re-centrifuged for $4 \mathrm{~min}$ at 10,170 rpm $(10,200 \mathrm{~g})$ to yield a supernatant, S2', and a pellet, $\mathrm{P} 2$ '. The supernatant S2' and S2 were combined, followed by centrifugation for $115 \mathrm{~min}$ in a Beckman 80Ti rotor (Beckman Instrument Co., CA, USA) at 42,000 rpm $(165,000 \mathrm{~g})$ to yield a pellet fraction P3 and a S3 (a cytosol fraction). The pellet $\mathrm{P} 2$ ' was re-suspended in buffered sucrose and was referred to a crude synaptosomal fraction. This suspension was homogenized, and the resulting P2'-lysate (L) was poured rapidly in 1-M HEPES-KOH buffer ( $\mathrm{pH} 7.4)$ on ice for $30 \mathrm{~min}$. It was then centrifuged for $10 \mathrm{~min}$ at $16,300 \mathrm{rpm}(25,000 \mathrm{~g})$ to yield a lysate pellet (LP1) and a lysate supernatant (LS1). The supernatant was collected and centrifuged for $115 \mathrm{~min}$ in a Beckman $80 \mathrm{Ti}$ rotor at $42,000 \mathrm{rpm}(165,000 \mathrm{~g})$. The supernatants (LS2) and the pellet (LP2) were pooled.

\section{WGA Purification of LP2 Fraction}

Synaptic vesicle fraction (LP2) from the hippocampus and the cerebral cortex was lysed with solubilization buffer A (20-mM HEPES (pH 7.4), 150-mM NaCl, 1-mM EDTA, $1 \%$ digitonin) and centrifuged at $22,000 \mathrm{~g}$ for $30 \mathrm{~min}$, and the pellet was lysed with solubilization buffer B (20-mM HEPES (pH 7.4), 150-mM NaCl, 1-mM EDTA, $0.5 \% n$ dodecyl- $\beta$-D-maltoside (DDM)). The supernatant, after adding 1-mM $\mathrm{CaCl}_{2}$ and $1-\mathrm{mM} \mathrm{MgCl}_{2}$, was loaded onto a wheat germ agglutinin (WGA) sepharose column and incubated overnight at $4{ }^{\circ} \mathrm{C}$. The first flow-through was collected and stored. The column was washed with washing buffer (20-mM HEPES (pH 7.4), 150-mM NaCl, 1-mM EDTA, $0.05 \% \mathrm{DDM})$. The binding proteins were eluted with the elution buffer ( $N$-acetylglucosamine $250 \mathrm{mM}$ in washing buffer). Equal amounts of protein lysates from the flowthrough and glycoprotein-enriched samples, after precipitating with ProteoExtract ${ }^{\circledR}$ (Calbiochem, Germany), were 
separated by SDS-PAGE and transferred to the PVDF membrane. Immunoreactive bands for $\beta$-dystroglycan and Dp40 were visualized using Super signal West pico.

Immunoaffinity Chromatography for LC-MS/MS Analyses

Protein $\mathrm{G}$ agarose beads cross-linked with the $\mathrm{N}$-term $\mathrm{Ab}$ by DMP (dimethyl pimelimidate-2 $\mathrm{HCl}$ ) were incubated with the WGA flow-through fraction overnight at $4^{\circ} \mathrm{C}$. The beads were put into the mini column and washed with washing buffer (20-mM HEPES(pH 7.4), 0.05\% DDM, 150-mM $\mathrm{NaCl}, 1-\mathrm{mM}$ EDTA). The binding fraction was eluted with peptide-containing elution buffer $(100 \mu \mathrm{g} / \mathrm{ml}$ of the antigen peptide, i.e., N-terminal 14 amino acids (MREQLKGHETQTTCW) specific to the short isoform dystrophin in washing buffer). The eluted fractions were precipitated using ProteoExtract ${ }^{\mathbb{R}}$ Protein precipitation kit (Merk, Japan). The dissolved pellet was separated by SDS-PAGE and stained by silver staining kit (Wako, Japan). The high-intensity protein bands were cut out from the gel, followed by LC-MS/MS analysis (Apro Life Science Institute Inc, Japan). The Mascot scores were obtained from Mascot searches (Matrix science), and the Mascot default significance threshold of $p<0.05$ for assignments was used in this study. The rest of the fractions were separated by SDS-PAGE and transferred to the PVDF membrane. Immunoreactive bands for Dp40, syntaxin1A, SNAP25, VAMP2, synaptotagmin, and CaMK II were visualized using ECL Western Blotting Detection System.

\section{Immunoprecipitation}

A Dp40-enriched LP2 fraction purified by WGA (300- $\mu \mathrm{g}$ protein) was preincubated with $50 \mu \mathrm{l}$ of anti-rabbit $\operatorname{IgG}$ beads (eBioscience, SanDiego, USA) for $30 \mathrm{~min}$ at $4^{\circ} \mathrm{C}$. Beads were eliminated by centrifugation, and extracts were incubated with $2-10-\mu \mathrm{g}$ immunoprecipitating antibody for $1 \mathrm{~h}$ at $4^{\circ} \mathrm{C}$. Fresh $50 \mu \mathrm{l}$ of IgG beads was added to the sample and incubated overnight at $4^{\circ} \mathrm{C}$. Immunoprecipitated beads were washed with washing buffer (20-mM HEPES (pH 7.4), 150-mM NaCl, 1-mM EDTA, 0.5\% DDM), solubilized in the sample buffer, and heated to $95^{\circ} \mathrm{C}$ for $10 \mathrm{~min}$. The proteins within the immunoprecipitated complexes were then separated by SDS-PAGE and identified by specific primary antibodies as described previously.

\section{Cell Culture of Hippocampal Neurons}

and Double Immunofluorescence Study

Primary hippocampal neurons were prepared as follows: hippocampi dissected from mouse brains at embryonic day 17.5 (E17.5) were dissociated and plated onto 12-mmdiameter glass coverslips precoated with poly-D-lysine and laminin at a density of $1 \times 10^{4} \mathrm{cells} / \mathrm{cm}^{2}$. Cultures were maintained in Neurobasal medium (Invitrogen, Japan) supplemented with 2\% B27 (GIBCO, Invitrogen, Japan) and 0.5-mML-glutamine (GIBCO, Invitrogen, Japan) as described elsewhere [17]. The hippocampal neurons were cultured for 10 days in 24-well plates and then fixed with acetone/ methanol (1:1) for $15 \mathrm{~min}$ on ice. After blocking, cells were sequentially incubated with the following primary antibodies overnight at $4^{\circ} \mathrm{C}$. At first, cells were incubated with $\mathrm{N}$-term $\mathrm{Ab}$ (dilution 1:100), followed by AlexaFluor546 ${ }^{\circledR}$-conjugated goat anti-rabbit IgG as secondary antibody. Subsequently, cells were incubated with Dys2 (dilution 1:100) and antisynaptic markers such as syntaxin1 (1:50), SNAP25 (1:1000), VAMP1/2 (1:100), synaptotagmin (1:500), CaMK II alpha (1:500), and PSD95 (1:500), respectively. Cells were incubated with secondary antibodies, AlexaFluor ${ }^{\circledR} 488$ conjugated goat anti-rabbit $\operatorname{IgG}$, and AlexaFluor ${ }^{\circledR} 647$ conjugated goat anti-mouse $\operatorname{IgG}$ at 1:1,000 for $1 \mathrm{~h}$. After washing, cells were mounted with SlowFade ${ }^{\circledR}$ (Invitrogen, Japan). The specimens were observed and photographed under a confocal laser microscope (FV-1000, OLYMPUS, Japan).

Image Analysis

In order to evaluate co-localization of DP-40 and presynaptic markers such as syntaxin1A, SNAP25, VAMP2, synaptotagmin, and synaptophysin as well as a postsynaptic marker, PSD 95, on neurites, we performed morphometrical analyses (Adobe photoshop CS53 EXTENDED ver 10.0.1) (Online Resource 3). We measured every pixel showing immunofluorescence after binarization of digital images and calculated ratios of target areas. Target areas included: singly immunoreactive for $\mathrm{N}$-term $\mathrm{Ab}$ (I, indicated by red fluorescence), C-terminal dystrophin, Dys2 (II, indicated by green fluorescence), and different synaptic markers (III, indicated by blue fluorescence); double immunoreactive for Dys2 and different synaptic markers (IV, indicated by light blue fluorescence), N-term $\mathrm{Ab}$ and different synaptic markers (V, indicated by pink fluorescence), and $\mathrm{N}$-term $\mathrm{Ab}$ and Dys2 (VI, indicated by yellow fluorescence); and triply immunoreactive for N-term Ab, Dys2, and different synaptic markers (VII, indicated by white fluorescence). The areas immunoreactive for both of $\mathrm{N}$-term $\mathrm{Ab}$ and different synaptic markers without immunoreactivity for Dys2 indicate presynaptic areas showing co-localization of DP-40 and presynaptic molecules.

\section{Results}

Confirmation of the Specificity of the Anti-N-terminal Specific Antibody (N-term $\mathrm{Ab}$ )

The rabbit polyclonal antibody was raised to the peptide carrying the N-terminal sequence common among Dp71, 
Dp58, and Dp40 proteins, and the specificity of the antibody was then evaluated. First, we performed the immunochemical detection of the recombinant GST with the antigen peptide sequence at the N-termini. The antibody successfully reacted with the antigen-fused GST but not GST itself (data not shown). The antibody was also reacted with the insect cell line-expressed recombinant mouse Dp40 as a 34.2-kDa band (Fig. 2a). In these experiments, the antigen absorption inhibited the detection of all of the recombinant proteins.

We detected immunochemically three short isoforms of dystrophin in adult mouse whole brain lysate; apparent molecular weight 69.4, 47.1, and $34.2 \mathrm{kDa}$, respectively (Fig. 2b). These major bands are thought to correspond to mouse Dp71, Dp58, and Dp40, respectively, because the molecular weight of the fastest migrating band is consistent with the one of the aforementioned recombinant Dp40 (Fig. 2a) [18, 19]. These proteins were not detected with

a

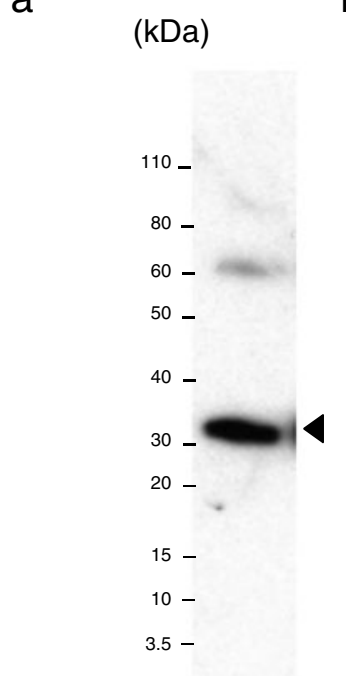

C

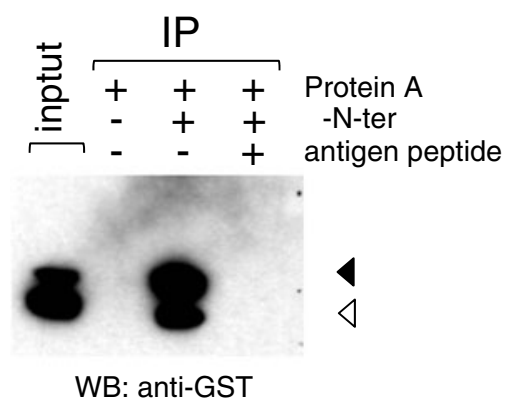

Fig. 2 Short isoforms of dystrophin were detected by western blot analyses and immunohistochemistry using the N-term $\mathrm{Ab}$. a The N-term $\mathrm{Ab}$ reacted with the insect cell line-expressed recombinant mouse $\mathrm{Dp} 40$ as a $34.2-\mathrm{kDa}$ band (arrowhead). b Three major bands were detected with apparent molecular weight $69.4,47.1$, and $34.2 \mathrm{kDa}$, respectively, in adult mouse whole brain lysate. c The GST-fused the antigen-preabsorbed antibody. The 69.4-kDa band was detected as two split bands $(69.4$ and $65.1 \mathrm{kDa})$ when the amount of loaded sample was adjusted. Two split bands of Dp71 were considered to correspond to the Dp71 isoforms with long or short C-terminal sequence that was produced by alternative splicing. The apparent $140.9-\mathrm{kDa}$ protein band (indicated by open arrowhead in Fig. 2b) was seldom reproducible. This band may correspond to another isoform Dp140 expressed in the brain because the preabsorbed antibody failed to detect it and because the C-terminal sequence of the antigen peptide is common among all isoforms of dystrophin. It is, however, noteworthy that this band was seldom detected reproducibly in western blot and never in the following affinity purification experiments. Taken together, these results suggest that the reactivity of our antibody is highly specific towards the three short isoforms of dystrophin.

d

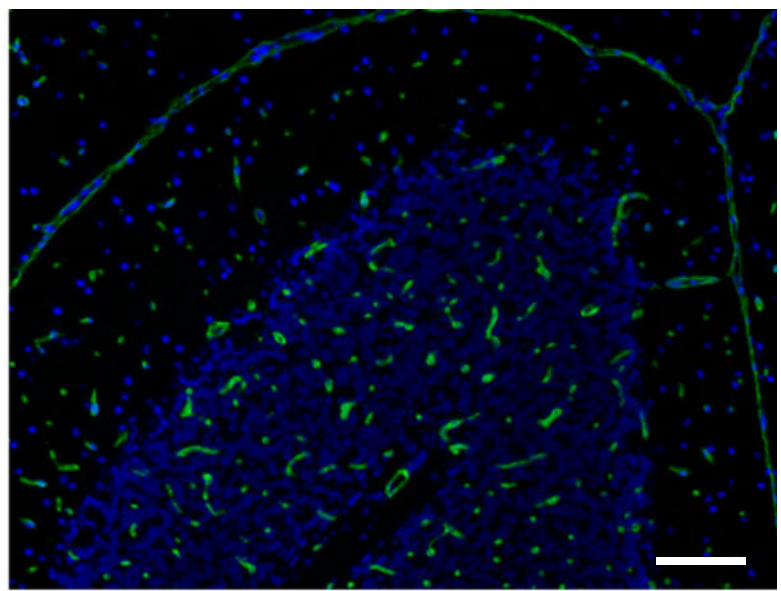

e

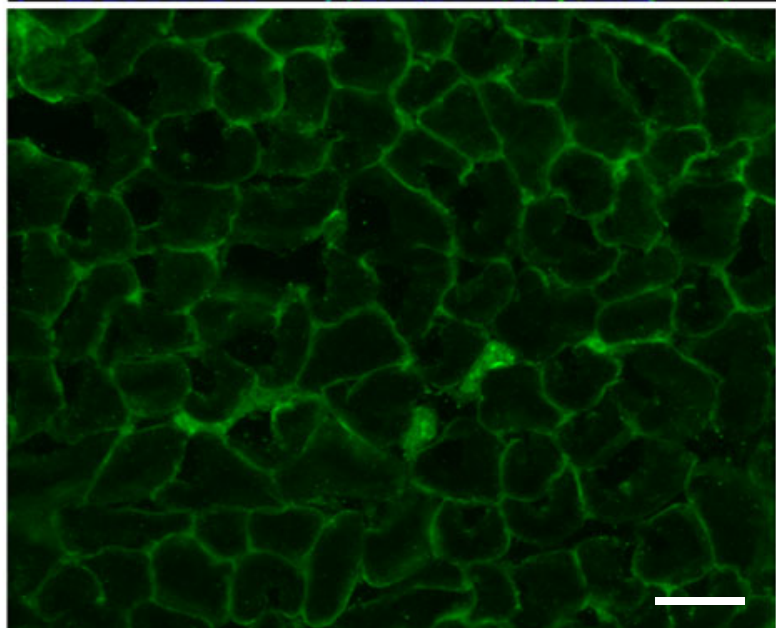

antigen protein but not GST itself was successfully precipitated with the $\mathrm{N}$-term Ab. d Immunoreactivity for short isoforms of dystrophin (green) was observed in the perivascular area and glia limitans, being composed of astrocytic foot processes, in the cerebellum. Scale bar $=50 \mu \mathrm{m}$. e Immunoreactivity for 427-kDa dystrophin (green) was localized in the sarcolemma of skeletal muscle. Scale bar $=25 \mu \mathrm{m}$ 
The antibody was also applicable to the immunoprecipitation of the short isoform proteins because the GST-fused antigen protein but not GST itself was successfully precipitated with the antibody. This reaction was prevented by the preabsorption with the peptide (Fig. 2c).

Immunofluorescent signals were observed in the perivascular astrocytic endfeet localized in the granular and molecular layer of the mouse cerebellum as well as in the glia limitans (Fig. 2d). This expression pattern was consistent with previous reports [20]. In skeletal muscles, dystrophin-immunofluorescence was localized in the sarcolemma, which showed that $\mathrm{N}$-term $\mathrm{Ab}$ recognized the $427-\mathrm{kDa}$ dystrophin of the muscle at the common regions of dystrophin (Fig. 2e).

Taken together, our antibody is considered to be highly specific towards three short isoforms of dystrophin, and it is thus applicable to immunoprecipitation experiments in addition to immunochemical and immunohistochemical detection.

Biochemical Characterization and Subcellular Localization of Dp40 in Mice Brain Extracts

Dp40 showed two distinct biochemical characteristics different from Dp58 and Dp71. The first was that Dp40 had a unique detergent solubility different from other short isoforms of dystrophin. Dp40 was insoluble in digitonin, although it is soluble in DDM and TritonX-100. On the other hand, Dp71 and Dp58 were soluble in all of the three detergents which we employed, including digitonin,
DDM, and TritonX-100 (Fig. 3a). Therefore, this distinct solubility in detergents allowed us to separate Dp40 from other two short isoforms of dystrophin. The second was that Dp40 showed a lower affinity to lectin, WGA. In order to analyze the interaction between Dp40 and glycosylated molecules so far known to form a dystrophin glycoprotein complex (DGC), we adopted WGA affinity column chromatography. It turned out that although both Dp71 and $\beta$-dystroglycan were detected in the WGA-bound fraction as reported previously [21], Dp40 was detectable in the WGA-unbound fraction (Fig. 3b). In addition, Dp58 was found in the WGA-bound fraction (data not shown). The low affinity between Dp40 and DGC led us to hypothesize that Dp40 might form a novel protein complex different from DGC. The DGC was already known to be composed of other short isoforms of dystrophin, which were abundantly expressed in neuronal cells, such as Dp71 and Dp58 [6]. As for subcellular localization of short isoforms of dystrophin, our studies revealed that Dp40 was enriched in the LP2 fraction (synaptic vesicle fraction) and Dp71 was present in similar membrane fractions, as previously described $[5,22]$. Dp58 was enriched in soluble fractions (Fig. 3c). We therefore decided to use the LP2 fraction to separate Dp40 and Dp71 from Dp58. Our procedure to obtain the Dp40-enriched protein extract was as follows: (1) After collecting the LP2 fraction of mouse cerebral cortex and hippocampus, the insoluble fractions of LP2 were eluted first with 1\% digitonin, followed by elution with $0.5 \%$ DDM, (2) the soluble DDM fractions were applied to WGA column, and the WGA-unbounded fractions were collected.
Fig. 3 Subcellular localization and biochemical characterization of Dp40 was demonstrated using the N-term Ab. a Dp40 was insoluble in $1 \%$ digitonin (Dig) and soluble in $0.5 \% \mathrm{DDM}$, as shown in Dig $\rightarrow$ DDM and 1\% Tx (TritonX-100; as shown in Dig $\leftrightarrow$ DDM $\rightarrow$ Tx). Dp71 and DP58 $(\mathrm{Dp} 71 \Delta 110)$ were soluble in digitonin, DDM, and Tx. b WGA affinity chromatography showed that Dp40 was detected in the WGA-unbound fraction (F.T flow-through) not in E1,E2, and $E 3$ ( $E$ elute; WGA-bound fraction). $W$ wash fraction. c Subcellular fractionation revealed that Dp40 and Dp71 were enriched in the $L P 2$ fraction (synaptic vesicle fraction), whereas DP58 was enriched in soluble fractions $(S 3)$

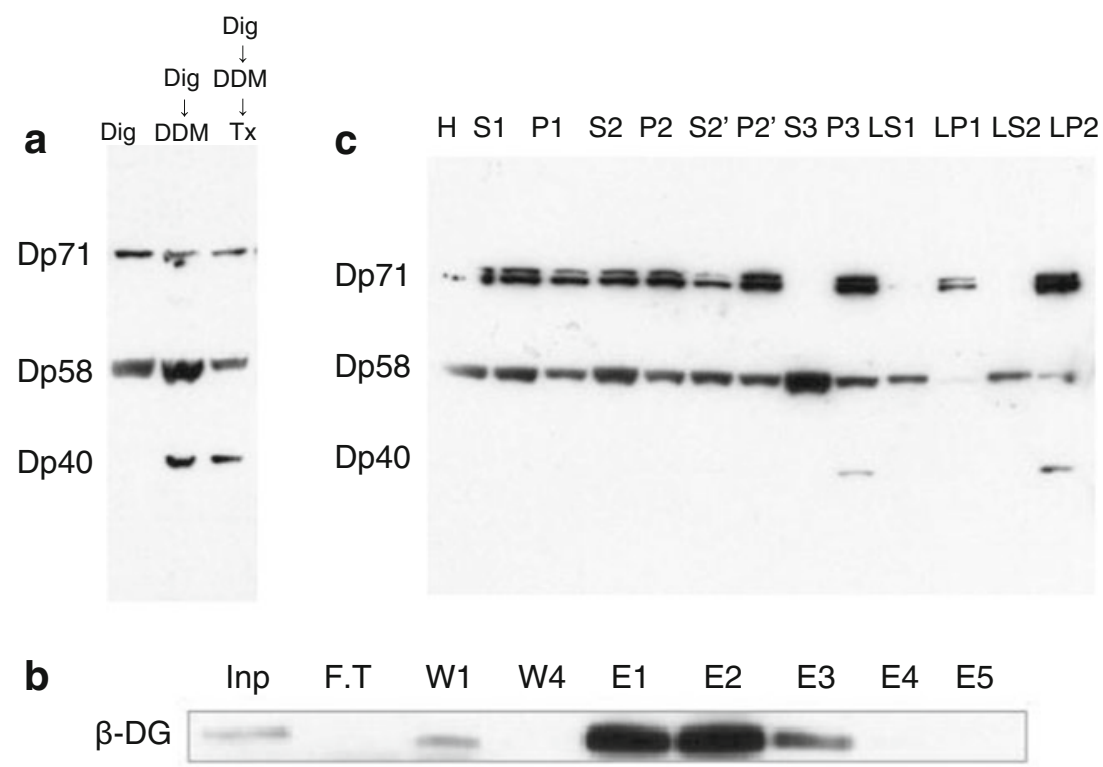

Dp40 
The Analysis of the Protein Complex Containing Dp40 by Immunoaffinity Column and LC-MS/MS

The Dp40-enriched fraction was applied to immunoaffinity column composed of protein $\mathrm{G}$ agarose beads cross-linked with the $\mathrm{N}$-term $\mathrm{Ab}$ against short isoforms of dystrophin, and bound fractions were then separated by SDS-PAGE. The intensely stained bands of smaller than $60 \mathrm{kDa}$ (indicated as $* 1, * 2, * 3$ in Fig. 4a) were cut and processed for the analysis with LC-MS/MS (Apro Life Science Institute Inc, Japan). Proteins identified by LC-MS/MS analysis were listed in Table 1. Syntaxin1A and SNAP25 (synaptosomeassociated protein of $25 \mathrm{kDa}$ ) were identified with high Mascot score, and the other vesicle trafficking proteins were also detected. Immunoblot analysis confirmed that Dp40 interacted with the SNARE complex, which included syntaxin1A, SNAP25, and VAMP2 (vesicle-associated membrane protein 2 , also called synaptobrevin). In addition, the other vesicle trafficking proteins, including synaptotagmin and CaMK II, were shown to interact with Dp40 (Fig. 4b).

\section{Co-immunoprecipitation with Presynaptic Proteins}

To validate the complex analysis described previously, Dp40-enriched extracts from LP2 fractions were subjected to immunoprecipitation studies with the N-term $\mathrm{Ab}$. It turned out that SNARE complex proteins such as syntaxin1A, SNAP25, and VAMP2 were co-immuoprecipitated with $\mathrm{Dp} 40$, but not with normal preimmune rabbit $\operatorname{IgG}$

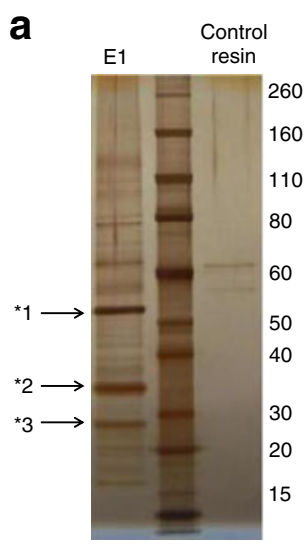

b

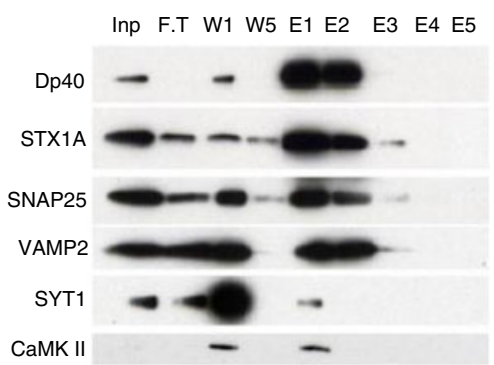

Fig. 4 Purification with affinity chromatography enabled the isolation of spots for LC-MS/MS, and immunoblot analysis revealed an interaction of Dp40 with the SNARE complex. a The elution samples were run on SDS-PAGE and stained with a Wako silver staining kit. Right lane is control (without treatment with the antibody). The bands $* 1,{ }^{*} 2$, and *3 were cut out for LC-MS/MS analysis. b Immunoblot analysis revealed that SNARE complex proteins such as syntaxin1A, SNAP25, and VAMP2 were detected and that other vesicle trafficking proteins, including synaptotagmin and CaMK II, were also detected with Dp40, although at lower levels
(Fig. 5a). Reciprocal immunoprecipitation studies revealed that syntaxin1A and SNAP25 interacted similarly with Dp40 in LP2 fractions (Fig. 5b), which suggests that Dp40 might play some functional role in the presynaptic terminal, including exocytotic vesicle docking or fusion with the plasma membrane.

For further analysis of the molecular components of the complex, Dp40-enriched fractions of LP2 were loaded onto a continuous $3-20 \%$ sucrose gradient and centrifuged for $16 \mathrm{~h}$ at $140,000 \mathrm{~g}$. Fourteen fractions were separately collected and analyzed by western blot (Online Resource 1A). In Dp40-enriched fraction, VAMP 2 was not enriched, and similarly Dp40 was not highly enriched in VAMP2-enriched fractions. The Dp40-enriched fraction was subjected to immunoprecipitation with the $\mathrm{N}$-term $\mathrm{Ab}$. It turned out that SNARE complex proteins such as syntaxin1A, SNAP25, and VAMP2 were co-immuoprecipitated with Dp40; however, reciprocal immunoprecipitation with VAMP2 demonstrated that VAMP2 did not interact with Dp40 (Online Resource 1B).

Co-localization of the Short Isoform Dystrophin with Presynaptic Proteins in Cultured Hippocampal Neurons

We studied whether Dp40 was co-localized with presynaptic proteins in primary cultured mouse hippocampal neurons using triple immunofluorescence techniques (Fig. 6). The immunofluorescence for the $\mathrm{N}$-term $\mathrm{Ab}$ showed a fine punctate pattern along the dendrites (red fluorescent signals, Fig. 6, Online Resource 2), which was consistent with previous reports [21]. Using confocal microscopy, we confirmed that some of the puncta exhibited co-localized immunostaining for the $\mathrm{N}$-term $\mathrm{Ab}$ (indicated by red fluorescence) and for syntaxin1A (blue fluorescence, Fig. 6a), SNAP25 (blue fluorescence, Fig. 6b), VAMP2 (blue fluorescence, Fig. 6c), CAMK II (blue fluorescence, Fig. 6d), synaptotagmin (blue fluorescence, Fig. 6e), and synaptophysin (blue fluorescence, Fig. 6f). It was thus concluded that the short isoform of dystrophin was expressed at some synaptic boutons present on both somata and axons. We could demonstrate that there were areas showing immunoreactivity for N-terminal short isoform of dystrophin and different synaptic markers and negative for Cterminal dystrophin, which indicated co-localization of DP40 and some synaptic molecules. The areas were $11.6 \pm$ $4.4 \%$ in syntaxin $1,15.0 \pm 4.1 \%$ in SNAP25, $23.7 \pm 6.9 \%$ in VAMP $1 / 2,12.7 \pm 6.6 \%$ in CaMK II, $8.7 \pm 10.6 \%$ in synaptotagmin, $6.4 \pm 3.7 \%$ in synaptophysin, and $7.7 \pm 6.0 \%$ in PSD95 (Online Resource 3). A few DP-40 puncta existed not only in the presynapse but also in the postsynapse. However, DP-40 was co-localized more specifically with presynaptic markers such as syntaxin1, SNAP25, and VAMP 
Table 1 Proteins identified by LC-MS/MS of excised and trypsin-digested gel bands

\begin{tabular}{|c|c|c|c|c|c|}
\hline Gel band & Accession no. & Description & Source & Mascot score & Unique peptide matched \\
\hline \multirow[t]{3}{*}{$* 1$} & gi 13542680 & Tublin, beta $2 \mathrm{C}$ & Mus musculus & 823 & 17 \\
\hline & gi 6755901 & Tublin, alpha 1 & Mus musculus & 783 & 15 \\
\hline & gi 39104626 & mKIAA0968 protein & Mus musculus & 269 & 5 \\
\hline \multirow[t]{3}{*}{$* 2$} & gi 15011853 & Syntaxin 1A & Mus musculus & 1,024 & 24 \\
\hline & gi 21746161 & Tublin, beta & Mus musculus & 193 & 4 \\
\hline & gi 14715019 & Syntaxin 12 & Mus musculus & 60 & 1 \\
\hline \multirow[t]{3}{*}{ *3 } & gi 6755588 & Synaptosomal-associated protein 25 & Mus musculus & 908 & 26 \\
\hline & gi 6679593 & Ras-related protein Rab-3A & Mus musculus & 99 & 1 \\
\hline & gi 4731936 & Synaotogyrin 3 & Mus musculus & 77 & 1 \\
\hline
\end{tabular}

$1 / 2$, being compatible with the results of immunoblots and LC-MS/MS.

\section{Discussion}

In the present study, we attempted to raise a polyclonal antibody against the N-terminal sequence common to three short isoforms of dystrophin and confirmed that the antibody actually recognized three isoforms, including Dp71, Dp58 (Dp71 1110$)$, and Dp40. It is worth noting that Dp40 was abundantly expressed in the brain and enriched in the synaptic vesicle fraction as assessed by subcellular fractionation. Furthermore, we demonstrated two biochemical characteristics of Dp40; firstly, Dp40 showed a detergent insolubility different from Dp71 and

a

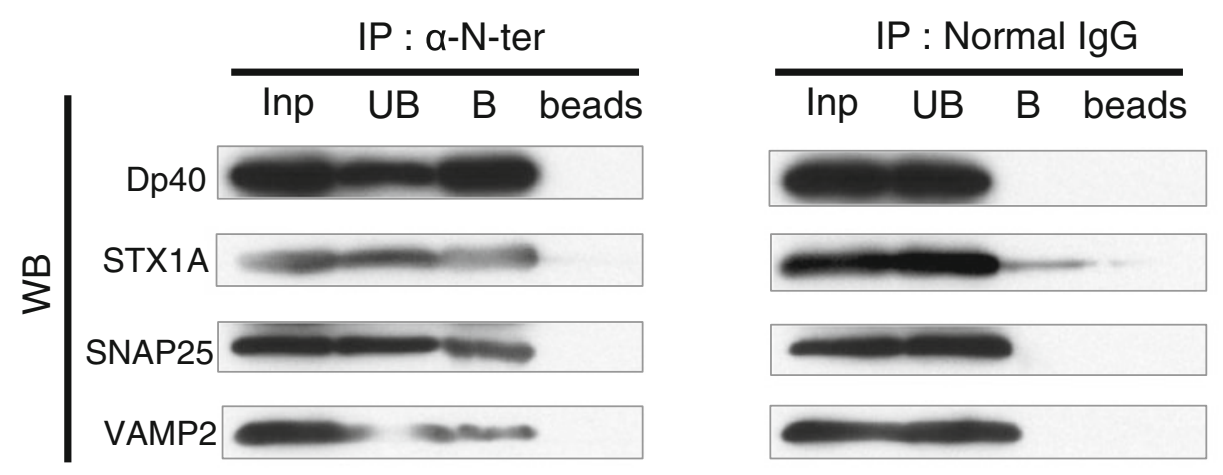

Reverse IP

b

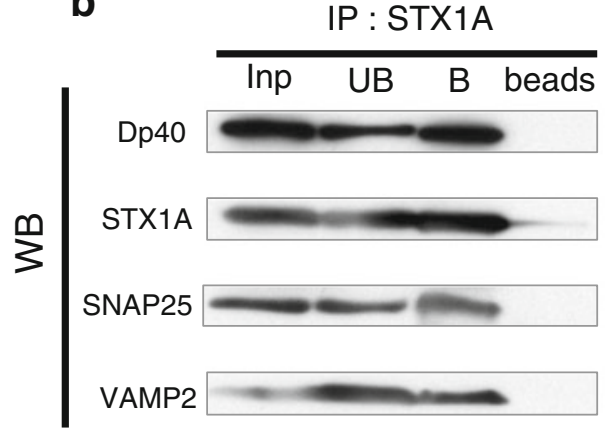

Fig. 5 The SNARE complex was co-immunoprecipitated with Dp40 when using the WGA-unbound LP2 fraction of brain lysate. a The lysate from Dp40-enriched LP2 fraction was immunoprecipitated with $\mathrm{N}$-term $\mathrm{Ab}$ or normal preimmune rabbit IgG. SNARE complex such as
syntaxin1A, SNAP25, and VAMP2 were co-immunoprecipitated by forward immunoprecipitation. b Same samples were used for reverse immunoprecipitation. Inp input, $U B$ unbound fraction, $B$ bound fraction 

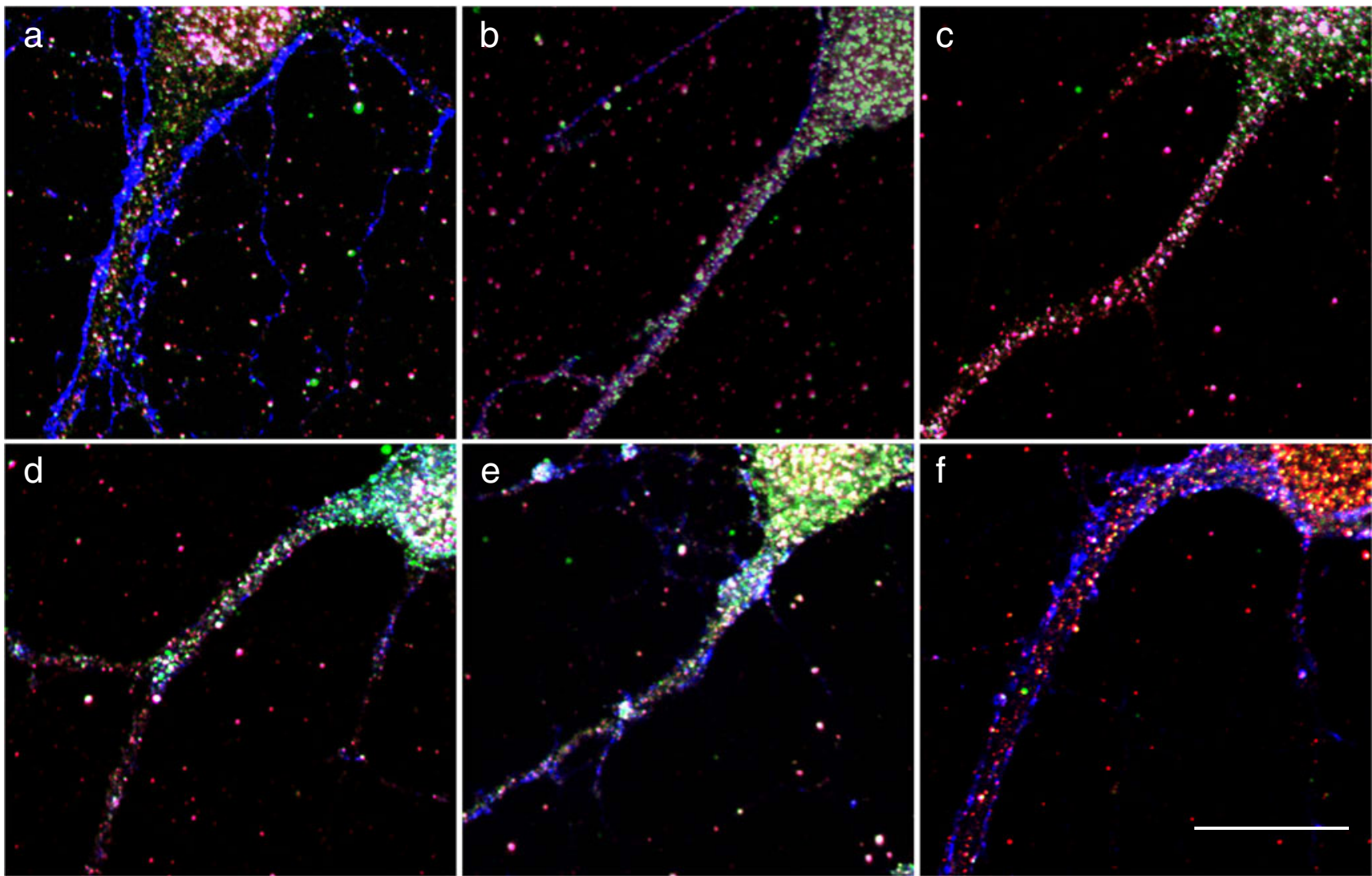

Fig. 6 Co-localization of short isoforms of dystrophin and presynaptic proteins in primary cultured mouse hippocampal neurons. Primary cultured hippocampal neurons were sequentially triply immunostained with the rabbit $\mathrm{N}$-term $\mathrm{Ab}(r e d)$, rabbit antibody against $\mathrm{C}$-terminal dystrophin (green), and with mouse antibodies against presynaptic proteins including syntaxin1 (a), SNAP25 (b), VAMP 1/2 (c), CaMK II (d), synaptotagmin (e), or synaptophysin (f) (blue). Short isoforms of dystrophin were focally co-localized with presynaptic proteins at apparent synaptic boutons (purple). Scale bar $=30 \mu \mathrm{m}$
Dp58, and secondly, Dp40 interacted poorly with $\beta$ dystroglycan which has been regarded as a main component of the DGC, while previous reports indicated that Dp71 and Dp58 interacted with $\beta$-dystroglycan at the cystein rich domain [23, 24]. These observations led us to hypothesize that Dp40 might form a novel protein complex different from DGC. Affinity chromatography and LC-MS/MS analysis revealed that the components of this protein complex with Dp40 were presynaptic proteins, such as syntaxin1A and SNAP25. To our knowledge, this is the first report to show that Dp40 interacted with a group of presynaptic proteins, and our observation should provide a new insight into understanding the function of Dp40 in the brain.

A possible relationship between the intellectual impairment and the altered expression of the C-terminal short isoforms of dystrophin in the brain has been discussed in previous case reports $[25,26]$. Some reports suggested that Dp71 deficiency was associated with mental retardation (MR) in a subpopulation of DMD patients [8, 10], and the deficiency was considered to contribute to the severity of the
MR phenotype. It was reported that patients with severe MR had mutations which influenced the expression not only of Dp71 but also of Dp40, although nothing was mentioned about the expression of Dp40 in those patients [10]. This observation prompted us to hypothesize that Dp40 might have novel functions in the brain which are relevant to cognitive function. Daoud et al. demonstrated that Dp71associated protein complexes interacted with specialized modular scaffolds that enabled glutamate receptors to cluster and to organize signaling cascades in postsynaptic densities [27]. They further showed that Dp71-null mice displayed abnormal synaptic organization and maturation in vivo, namely, abnormally enhanced glutamate transmission and altered synaptic plasticity in the CA1 area of the hippocampus. Since Dp71-null mice were deficient in not only Dp71 but Dp40 [28], the alteration of glutamate release might be caused by loss of both $\mathrm{Dp} 71$ and Dp40. Our present investigation clearly demonstrates that Dp40 has an interaction with a group of presynaptic proteins, such as syntaxin1A and SNAP25. Thus, it would be reasonable to expect that Dp40 may play a 
crucial role in presynaptic function because syntaxin1A and SNAP25 are involved in regulating exocytosis of synaptic vesicles in neurons. It is worth noting that ablation of syntaxin1 $\mathrm{A}$ in mice induced abnormal behavior by disrupting serotonergic systems [29].

At present, some issues still remain to be resolved. Although our analysis by affinity chromatography showed that Dp40 mainly interacted with SNARE (soluble N-ethyl maleimide sensitive factor attachment protein receptors) complex proteins such as syntaxin1A, SNAP25, and VAMP2, the reciprocal immunoprecipitation failed to demonstrate that VAMP2 did interact with Dp40. These paradoxical results might be due either to the possibility that the epitope of VAMP2 recognized by anti-VAMP2 antibody may overlap with or be in close proximity to the site that interacts to Dp40 or, alternatively, that the fraction of VAMP2, showing close association with Dp40, was below the sensitivity for detection. However, we clearly demonstrated with immunohistochemistry that VAMP2 was co-localized at least in part with short isoforms of dystrophin, including Dp40, when examined in cultured hippocampal neurons. The currently established concept of the SNARE complex includes VAMP2 [15]. Taken together, we thus concluded that VAMP2 was a candidate protein which interacted with Dp40 in the presynaptic site. But, there still remains some possibility that Dp40 protein complex exists as a different pool from the SNARE complex in the presynapse. Ohyama et al reported that CaMK II interacted with syntaxin1A and SNAP25 but not with VAMP2 [30]. Our results that CaMK II was detectable as an interacting molecule with Dp40 by LC-MS/MS and immunoblot analyses may also support their notion. The prominent nuclear staining was found in hippocampal neurons with both the N-term- and C-term- antibodies (ESM 2), which might be reactive to short isoforms of dystrophin such as Dp58 and Dp71. As nuclear localization and nuclear function have been already described for Dp71 [31,32], it is pertinent to take into account that Dp40 might have nuclear localization and a putative nuclear function.

The function of other brain dystrophin isoforms (Dp427, Dp140) has as yet been fully understood, although loss of those isoforms were reported to be associated with cognitive impairment [33, 34]. However, at present, it is difficult to conclude which defect, the defects in full-length dystrophin and/or in the shorter isoforms, may contribute to the development of cognitive impairment. It is worth stating that SNAREs are not only involved in steps of docking and fusion of synaptic vesicles to the active zone, but that they also play an important role in the $\mathrm{Ca}^{2+}$-triggering step itself [35]. Further experiments are required to address the important question of the molecular mechanisms of cognitive impairment in DMD patients.
In conclusion, we have shown here the expression and the protein interactions of the shortest isoform of dystrophin $\mathrm{Dp} 40$ in the mouse brain and demonstrated that Dp40 is a novel molecule assembling a group of presynaptic proteins especially involved in exocytosis. In order to better understand the functions of Dp40 in the brain, our laboratories are currently studying with siRNA silencing as well as Dp40 conditional knockout mice.

Acknowledgments The authors are grateful to Prof. Dr. Lynn Landmesser, Chairman of the Department of Neurosciences, Case Western Reserve University, for her helpful comments on the manuscript. We are also grateful to Prof. Hajime Mori for providing us with the insect cell line-expressed recombinant mouse Dp40. This study was supported in part by a Grant-in-Aid for Young Scientists (to ST) from the Ministry of Education, Culture, Sports, Science and Technology, Japan and by Project Research Grants from Kyoto Prefectural University of Medicine (to HD, KI, SF).

Open Access This article is distributed under the terms of the Creative Commons Attribution Noncommercial License which permits any noncommercial use, distribution, and reproduction in any medium, provided the original author(s) and source are credited.

\section{References}

1. Emery AEH (1987) Central nervous system. In: Motulsky AG (ed) Duchenne muscular dystrophy 2. Oxford University Press, New York, p 99

2. Bresolin N, Castelli E, Comi GP et al (1994) Cognitive impairment in Duchenne muscular dystrophy. Neuromuscul Disord 4:359-369

3. Wicksell RK, Kihlgren M, Melin L, Eeg-Olofsson O (2004) Specific cognitive deficits are common in children with Duchenne muscular dystrophy. Dev Med Child Neurol 46:154-159

4. Billard C, Gillet P, Signoret JL et al (1992) Cognitive functions in Duchenne muscular dystrophy: a reappraisal and comparison with spinal muscular atrophy. Neuromuscul Disord 2:371-378

5. Lederfein D, Levy Z, Augier N et al (1992) A 71-kilodalton protein is a major product of the Duchenne muscular dystrophy gene in brain and other nonmuscle tissues. Proc Natl Acad Sci USA 89:5346-5350

6. Tadayoni R, Renden A, Soria-Jasso LE, Cisneros B (2011) Dystrophin Dp71: the smallest but multifunctional product of the Duchenne muscular dystrophy gene. Mol Neurobiol. doi:10.1007/s12035-011-8218-9

7. Dystrophin isoforms: Leiden muscular dystrophy Pages ${ }^{\mathcal{C}}$

8. Moizard MP, Toutain A, Fournier D et al (2000) Severe cognitive impairment in DMD: obvious clinical indication for Dp71 isoform point mutation screening. Eur J Hum Genet 8:552-556

9. Lidov HG (1996) Dystrophin in the nervous system. Brain Pathol 6:63-77

10. Daoud F, Angeard N, Demerre B et al (2009) Analysis of Dp71 contribution in the severity of mental retardation through comparison of Duchenne and Becker patients differing by mutation consequences on Dp71 expression. Hum Mol Genet 18:3779-3794

11. Desguerre I, Christov C, Mayer M et al (2009) Clinical heterogeneity of Duchenne muscular dystrophy (DMD): definition of subphenotypes and predictive criteria by long-term follow-up. PLoS ONE 4:e4347 
12. Tinsley JM, Blake DJ, Davies KE (1993) Apo-dystrophin-3: a $2.2 \mathrm{~kb}$ transcript from the DMD locus encoding the dystrophin glycoprotein binding site. Hum Mol Genet 2:521-524

13. Bennett MK, Garćia-Arrarás JE, Elferink LA et al (1993) The syntaxin family of vesicular transport receptors. Cell 74:863873

14. Südhof TC (1995) The synaptic vesicle cycle: a cascade of protein-protein interactions. Nature 375:645-653

15. Jahn R, Hanson PI (1998) Membrane fusion. SNAREs line up in new environment. Nature 393:14-15

16. Huttner WB, Schiebler W, Greengard P, De Camilli P (1983) Synapsin I (protein I), a nerve terminal-specific phosphoprotein. III. Its association with synaptic vesicles studied in a highly purified synaptic vesicle preparation. J Cell Biol 96:13741388

17. Brewer GJ, Torricelli JR, Evege EK, Price PJ (1993) Optimized survival of hippocampal neurons in B27-supplemented Neurobasal, a new serum-free medium combination. J Neurosci Res 35:567-576

18. Austin RC, Morris GE, Howard PL, Klamut HJ, Ray PN (2000) Expression and synthesis of alternatively spliced variants of Dp71 in adult human brain. Neuromuscul Disord 10:187-193

19. Austin RC, Fox JE, Werstuck GH et al (2002) Identification of Dp71 isoforms in the platelet membrane cytoskeleton. Potential role in thrombin-mediated platelet adhesion. J Biol Chem 277: 47106-47113

20. Nicchia GP, Rossi A, Nudel U, Svelto M, Frigeri A (2008) Dystrophindependent and -independent AQP4 pools are expressed in the mouse brain. Glia 56:869-876

21. Nicchia GP, Cogotzi L, Rossi A et al (2008) Expression of multiple AQP4 pools in the plasma membrane and their association with the dystrophin complex. J Neurochem 105:2156-2165

22. Jung D, Filliol D, Metz-Boutigue MH, Rendon A (1993) Characterization and subcellular localization of the dystrophin-protein 71 (Dp71) from brain. Neuromuscul Disord 3:515-518

23. Cox GA, Sunada Y, Campbell KP, Chamberlain JS (1994) Dp71 can restore the dystrophin-associated glycoprotein complex in muscle but fails to prevent dystrophy. Nat Genet 8:333-339

24. Greenberg DS, Schatz Y, Levy Z, Pizzo P, Yaffe D, Nudel U (1996) Reduced levels of dystrophin associated proteins in the brains of mice deficient for Dp71. Hum Mol Genet 5:12991303
25. Taylor PJ, Betts GA, Maroulis S et al (2010) Dystrophin gene mutation location and the risk of cognitive impairment in Duchenne muscular dystrophy. PloS ONE 5:e8803

26. Lenk U, Hanke R, Thiele H, Speer A (1993) Point mutations at the carboxy terminus of the human dystrophin gene: implications for an association with mental retardation in DMD patients. Hum Mol Genet 2:1877-1881

27. Daoud F, Candelario-Martínez A, Billard J-M et al (2009) Role of mental retardation-associated dystrophin-gene product Dp71 in excitatory synapse organization, synaptic plasticity and behavioral function. PloS ONE 4:e6574

28. Sarig R, Mezger-Lallemand V, Gitelman I et al (1999) Targeted inactivation of $\mathrm{Dp} 71$, the major non-muscle product of the DMD gene: differential activity of the Dp71 promoter during development. Hum Mol Genet 8:1-10

29. Fujiwara T, Snada M, Kofuji T, Yoshikawa T, Akagawa K (2010) HPC/syntaxin 1A gene knockout mice show abnormal behavior possibly related to a disruption in 5-HTergic systems. Eur J Neurosci 32:99-107

30. Ohyama A, Hosaka K, Komiya Y et al (2002) Regulation of exocytosis through Ca2+/ATP-dependent binding of autophosphorylated $\mathrm{Ca} 2+/$ calmodulin-activated protein kinase II to syntaxin1A. J Neurosci 22:3342-3351

31. Villarreal-Silva M, Suárez-Sánchez R, Rodrígeuz-Muñoz R, Mornet D, Cisneros B (2010) Dystrophin Dp71 is critical for stability of the DAPs in the nucleus of PC12 cells. Neurochem Res 35:366-373

32. Villarreal-Silva M, Centeno-Cruz FM, Suárez-Sánchez R, Garrido E, Cisneros B (2011) Knockdown of dystrophin Dp71 impairs PC12 cells cycle: localization in the spindle and cytokinesis structures implies a role for Dp71 in cell division. PLoS ONE 6(8):e23504. doi:10.1371/journal.pone.0023504

33. Moizard MP, Billard C, Toutain A, Berret F, Marmin N, Moraine C (1998) Are Dp71 and Dp140 brain dystrophin isoforms related to cognitive impairment in Duchenne muscular dystrophy? Am J Med Genet 80:32-41

34. Vaillend C, Billard JM, Claudepierre T, Rendon A, Dutar P, Ungerer A (1998) Spatial discrimination learning and CA1 hippocampal synaptic plasticity in $\mathrm{mdx}$ and $\mathrm{mdx} 3 \mathrm{cv}$ mice lacking dystrophin gene products. Neuroscience 86:53-66

35. Sakaba T, Stein A, Jahn R, Neher E (2005) Distinct kinetic changes in neurotransmitter release after SNARE protein cleavage. Science 309:491-494 